\title{
Respostas de estresse em pirarucu (Arapaima gigas) durante práticas de rotina em piscicultura
}

\author{
Franmir Rodrigues BRANDÃO ${ }^{1}$, Levy de Carvalho GOMES ${ }^{2,3}$, Edsandra Campos CHAGAS²
}

\section{RESUMO}

O pirarucu é um peixe nativo da bacia Amazônica com respiração aérea obrigatória. Em condições de criação, atinge até $10 \mathrm{~kg}$ em um ano, sendo um dos peixes com maior potencial para criação na Amazônia. O objetivo deste estudo foi avaliar as respostas de estresse em pirarucu quando submetido a práticas comuns em sistema de criação. Para isso, foram realizados três diferentes experimentos: 1) transporte; 2) adensamento; e 3) exposição à amônia. Foram analisados parâmetros do metabolismo energético (glicose e lactato), hormonal (cortisol), e de hematologia (hematócrito). Em todos os protocolos testados foram observadas alteraçôes nos parâmetros fisiológicos do pirarucu. As respostas de estresse durante o transporte foram similares às do adensamento, porém, a magnitude das repostas ao adensamento foi maior. A exposição à amônia não causou alteração imediata nos parâmetros fisiológicos, havendo latência nas respostas de estresse. Com os resultados obtidos, pode-se concluir que as alteraçóes nos parâmetros metabólicos ocorrem no momento de maior intensidade de manejo, e provavelmente podem ser reduzidas com adoção de boas práticas na criação.

\section{PALAVRAS-CHAVE}

indicador de estresse, glicose, cortisol, lactato, pirarucu

\section{Stress responses of pirarucu (Arapaima gigas) during routine aquaculture practices}

\begin{abstract}
Pirarucu is an obligatory air breathing fish native to the Amazon basin. In rearing conditions it reaches up to $10 \mathrm{~kg}$ in one year, being one of the fish with the greatest potential for farming in the Amazon. The aim of this study was to verify stress responses in pirarucu during routine aquaculture practices. Three experiments were carried out: 1) transport; 2) crowding; and 3) exposure to ammonia. Parameters of the energy metabolism (glucose and lactate), hormonal (cortisol), and hematological (hematocrit) were analyzed. All experiments caused alterations in the physiological parameters of pirarucu. The stress responses during transport are similar to crowding. However, the magnitude of the responses to crowding was higher. Ammonia exposure does not cause an immediate alteration in the physiological parameters, with a latency period in the stress responses. From the observed results it is possible to conclude that alterations in the metabolic parameters occur during the more intense management periods and probably can be prevented adopting best management practices.
\end{abstract}

\section{KEY WORDS}

stress indicator, glucose, cortisol, lactate, pirarucu

\footnotetext{
1 Programa de capacitação, Embrapa Amazônia Ocidental, Caixa Postal 319, CEP 69011-970, Manaus, AM.

${ }^{2}$ Embrapa Amazônia Ocidental, Caixa Postal 319, CEP 69011-970, Manaus, AM, Brasil.

${ }^{3}$ Autor para correspondência, e-mail: levy@cpaa.embrapa.br. Endereço atual: Departamento de Ecologia e Recursos Naturais, Universidade Federal do Espírito Santo. Av. Fernando Ferrari, 514, 29075-900, Vitória, ES.
} 


\section{INTRODUÇÃO}

O pirarucu (Arapaima gigas), peixe da família Arapaimidae (Ferraris, 2003), é nativo da bacia Amazônica e apresenta respiração aérea obrigatória facilitando sua criação em ambientes com baixa disponibilidade de oxigênio (Salvo-Souza \& Val, 1990). O pirarucu suporta altas densidades de estocagem (Cavero et al., 2003) e sua carne tem ótima aceitação no mercado, o que permite alcançar bom preço de venda. É a espécie mais promissora para o desenvolvimento da piscicultura em regime intensivo na região Amazônica. Possui alta taxa de crescimento, podendo alcançar de 7 a $10 \mathrm{~kg}$ no primeiro ano de criação (Imbiriba, 2001; Pereira-Filho et al., 2003).

$\mathrm{O}$ transporte, o adensamento e a exposição a condiçōes extremas de qualidade de água são atualmente alguns dos principais problemas em estações de piscicultura, por causarem grande estresse nos peixes (Wedemeyer, 1996). Durante o transporte e o adensamento, o principal precursor do estresse é a abrasão mecânica causada pelo inevitável contato entre os peixes quando a densidade é elevada (Ross \& Ross, 1999; Gomes et al., 2003a). Os peixes respondem ao estresse de forma a refletir a severidade e a duração do estressor (Barton, 1997). Estas respostas preparam o organismo para a chamada luta e fuga, ou seja, a tentativa de escapar da adversidade, e podem variar de acordo com a intensidade e duração do agente estressor (Morgan \& Iwama, 1997; Urbinati et al., 2004). Conseqüentemente, as reações fisiológicas dos peixes a esses tipos agudos de estresse necessitam ser analisadas, tanto em relação ao tipo de resposta, como à sua intensidade (Krieger-Azolini et al., 1989).

As respostas de estresse são divididas em três categorias: primária, secundária e terciária (Barton, 1997; Barton et al., 2002). As respostas primárias são as hormonais, as secundárias são mudanças nos parâmetros fisiológicos e bioquímicos e as terciárias são o comprometimento no desempenho, mudanças no comportamento e aumento da suscetibilidade a doenças. $\mathrm{O}$ cortisol, principal corticosteróide em peixes, é considerado um bom indicador para avaliação de estresse primário (Mommsen $e t$ al., 1999). Os resultados de cortisol podem ser facilmente comparados com outras espécies, uma vez que existe vasta literatura sobre este indicador. Um bom indicador para resposta secundária é a glicose do sangue ou plasma, pois esta avaliação pode ser realizada na criação, com medidores de glicose de simples utilização e facilmente encontrados no mercado (Wells \& Pankhurst, 1999). O lactato também é um bom indicador de estresse, e indica o acúmulo de ácido láctico decorrente do aumento do exercício físico à medida que os animais são expostos a um estressor (Barton et al., 2002; Morgan \& Iwama, 1997).

As respostas de estresse do pirarucu constituem uma importante ferramenta para formular um procedimento de boas práticas de manejo, para que o peixe seja manuseado de forma a não comprometer seu desenvolvimento no sistema de criação. Portanto, o objetivo deste trabalho foi analisar as respostas de estresse em pirarucu quando submetido a práticas comuns em sistema de criação, como o transporte, o adensamento e a exposição a altas concentraçóes de amônia.

\section{MATERIAL E MÉTODOS}

Os protocolos experimentais utilizados seguiram roteiro de trabalhos previamente publicados, que trataram de questôes semelhantes com pirarucu ou outras espécies de peixes (Barnnet \& Pankhurst, 1998; Barton et al., 2000; Barton, 2000; Cavero et al., 2004; Gomes et al., 2003b). Este trabalho foi realizado na estação de piscicultura da Embrapa Amazônia Ocidental.

\section{EXPERIMENTO I - TRANSPORTE}

Os juvenis de pirarucu ( $\mathrm{n}=54$; peso médio $32,79 \pm 2,35 \mathrm{~g}$ ), previamente treinados a aceitar ração, foram aclimatados em um viveiro de $200 \mathrm{~m}^{3}$ por 20 dias, onde receberam ração extrusada para peixes carnívoros com $45 \%$ de proteína bruta. Após a aclimatação, os peixes foram transferidos, por 24 horas, para uma caixa de depuração com capacidade para 2000L, com fluxo de água constante. $\mathrm{O}$ transporte foi realizado em sacos plásticos (3 repetiçôes), com capacidade para 60L, nos quais foram adicionados $20 \mathrm{~L}$ de água e posteriormente oxigênio puro. Foram transportados 12 peixes em cada saco plástico. $\mathrm{O}$ transporte foi realizado por rodovia durante três horas. Após o transporte, os peixes foram distribuídos igualmente em três tanques-rede de $1 \mathrm{~m}^{3}$ ( 1 tanque por repetição) onde permaneceram por 96 horas, para realizar amostragens periódicas.

Para avaliação dos parâmetros fisiológicos foi retirado sangue dos peixes nos seguintes momentos: antes do distúrbio no viveiro (AD; controle); antes do transporte (AT; na caixa de depuração); depois do transporte (DT; logo após a abertura do saco de transporte) e 24, 48 e 96 horas depois do transporte (24DT, 48DT e 96DT; no tanque-rede onde os peixes foram colocados após o transporte). Em cada momento, foram coletados três peixes de cada repetição, totalizando nove peixes por amostragem. Os peixes, após serem amostrados, eram descartados, não retornando para as unidades experimentais.

\section{EXPERIMENTO $\|$ - ADENSAMENTO}

Juvenis de pirarucu $(n=54$; peso médio $37,22 \pm 6,26 \mathrm{~g})$ foram igualmente estocados em três caixas de fibra de $2000 \mathrm{~L}$, com troca de água constante. Nestas caixas, os peixes foram mantidos por uma semana para aclimatação, sendo alimentados diariamente com ração comercial para peixes carnívoros com $45 \%$ de proteína bruta. Após este período, o volume de água da caixa foi reduzido até atingir 5\% do volume total, permanecendo assim durante 30 minutos, para simular um adensamento comumente obtido durante operações de captura. Logo após 30 minutos de adensamento, o volume de água retornou ao seu nível normal. Para avaliação dos parâmetros fisiológicos, foi retirado sangue dos peixes nos seguintes momentos: antes do adensamento (AA; controle); depois do adensamento (DA) e 6, 12, 24 e 48 horas 
depois do adensamento (6DA, 12DA, 24DA e 48DA). Em cada momento, foram amostrados três peixes de cada caixa, totalizando nove peixes por amostragem. Os peixes, após serem amostrados, eram descartados, não retornando para as unidades experimentais.

\section{EXPERIMENTO III - EXPOSIÇÃO A AMÔNIA.}

Os peixes $(n=45$, peso médio $39,35 \pm 2,82 \mathrm{~g})$ foram igualmente distribuídos em 3 caixas de fibra de 2000L. Nestas caixas, os peixes foram mantidos por uma semana para aclimatação, sendo alimentados diariamente com ração comercial para peixes carnívoros com $45 \%$ de proteína bruta. Após a aclimatação, foi colocado sulfato de amônia nas caixas até atingir a concentração de $20 \mathrm{mg}$ de amônia total/L, com pH mantido em 7. Esta concentração é letal para maioria das espécies de peixe, porém estudos recentes mostraram que o pirarucu resiste a esta concentração (Cavero et al., 2004). Esta concentração foi mantida por 24 horas. Logo após o período de exposição a água foi rapidamente trocada. Para avaliação dos parâmetros fisiológicos foi retirado sangue dos peixes nos seguintes momentos: antes $\mathrm{da}$ exposição (AE; controle); depois da exposição (DE) e 24, 48 e 96 horas depois da exposição (24DE, 48DE e 96DE). Em cada momento foram coletados três peixes de cada caixa, totalizando nove peixes por amostragem.

\section{ANÁLISES FISIOLÓGICAS}

A avaliação do perfil fisiológico dos peixes nos três experimentos foi realizada por meio dos seguintes indicadores: cortisol $(\mathrm{ng} / \mathrm{mL})$, glicose $(\mathrm{mg} / \mathrm{dL})$, lactato $(\mathrm{mmol} / \mathrm{L})$ e hematócrito (\%). O sangue dos peixes foi retirado por punção caudal com auxílio de seringas heparinizadas e o plasma separado por centrifugação (3.000 rpm, 10 minutos). O cortisol foi determinado pela técnica de imunoensaio enzimático por competição (EIA, Kit 55050, Human ${ }^{\circledR}$ ). A glicose e o lactato sanguíneo foram medidos em leitores digitais Advantage ${ }^{\mathrm{TM}} \mathrm{e}$ Arkray Factory ${ }^{\mathrm{TM}}$, respectivamente. O hematócrito foi determinado após centrifugação do sangue $(12.000 \mathrm{rpm}, 10$ minutos) em tubos microcapilares heparinizados.

\section{ANÁLISE ESTATÍSTICA}

Os resultados obtidos foram expressos em média \pm erro padrão. As médias obtidas nos diferentes tempos de amostragem foram comparadas com o momento controle (antes do distúrbio) por uma análise de variância (ANOVA) e teste de Dunnett a 5\% de probabilidade.

\section{RESULTADOS E DISCUSSÃO}

\section{EXPERIMENTO I - TRANSPORTE}

Gomes (2002) cita que embora o transporte seja uma das operaçôes mais importantes da piscicultura, pouca atenção tem sido dada ao assunto no Brasil. O transporte como prática de manejo em piscicultura intensiva pode ter duração variada dependendo da finalidade. Os peixes vivos são transportados para diversos destinos, incluindo a indústria e os estabelecimentos voltados à pesca esportiva, no caso de peixes adultos, e estabelecimentos de criação de engorda, no caso de larvas e juvenis. Em todos os casos, os animais devem chegar em boas condiçōes fisiológicas para satisfazer os critérios exigidos pelo comprador (Urbinati \& Carneiro, 2004).

Não houve mortalidade durante o período de transporte e até 96 horas após. O cortisol apresentou um aumento significativo no tempo $24 \mathrm{DT}(35,8 \pm 7,1 \mathrm{ng} / \mathrm{mL})$, quando comparado ao controle $(8,7 \pm 3,7 \mathrm{ng} / \mathrm{mL})$. Contudo, não foi observada elevação do cortisol nos momentos mais críticos do transporte, como AT e DT (Figura 1a), sendo este resultado semelhante ao obtido por Gomes et al. (2003b), durante o transporte de pirarucu de $1 \mathrm{~kg}$. Os resultados mostram uma grande latência na elevação do cortisol, sendo contrário aos resultados obtidos com outras espécies amazônicas como o tambaqui (Gomes et al., 2003a) e o matrinxã (Carneiro \& Urbinati, 2001), que apresentam elevação no cortisol logo após o transporte.

A glicose sanguínea apresentou diferença significativa após a captura/depuração (AT) e depois do transporte (DT), no momento mais intenso de manejo (Figura $1 \mathrm{~b}$ ). Os valores mais altos de glicose $(88,9 \pm 10,2 \mathrm{mg} / \mathrm{dL})$ foram obtidos no tempo DT. Esta elevação imediata da glicose em resposta ao estressor deve ter sido ocasionada por estímulo das catecolaminas, especialmente a epinefrina, que estimula a glicogenólise, ou seja, a transformação de glicogênio em glicose. A glicose sanguínea voltou para valores semelhantes ao AT no momento 24DT. Todas estas respostas visam manter uma quantidade de energia necessária para a manutenção das atividades metabólicas normais, e em caso de fuga, suprir a demanda excessiva dos músculos (Brinn, 2003).

O lactato apresentou uma diminuição significativa logo após o transporte quando comparado ao controle (Figura 1c). Os valores iniciais (AT) do pirarucu são altos quando comparados a outros peixes, e decorrentes da intensa natação do cardume no tanque de criação. Desta forma, os baixos valores encontrados no momento DT $(0,9 \pm 0,3 \mathrm{mmol} / \mathrm{L})$ demonstram que o peixe estava sob situação de estresse, como resposta direta ao pouco espaço disponível e a incapacidade de natação. Depois do transporte, quando retornaram para um ambiente com maior capacidade de natação, os peixes voltaram e exibir os valores iniciais de lactato. O salmão do Atlântico (Salmo salar) apresenta resposta diferente, os valores de lactato aumentam após o transporte e permanecem altos por até 48 horas (Iversen et al., 1998). A principal explicação para esta diferença é o sistema de transporte. Neste trabalho o sistema foi fechado, em sacos plástico. No experimento de Iversen et al. (1998), o sistema de transporte foi aberto, em caixa, onde o peixe apresenta natação ativa, acumulando assim, mais lactato.

O hematócrito apresentou um aumento significativo no tempo 24DT (Figura 1d), havendo uma grande latência na 

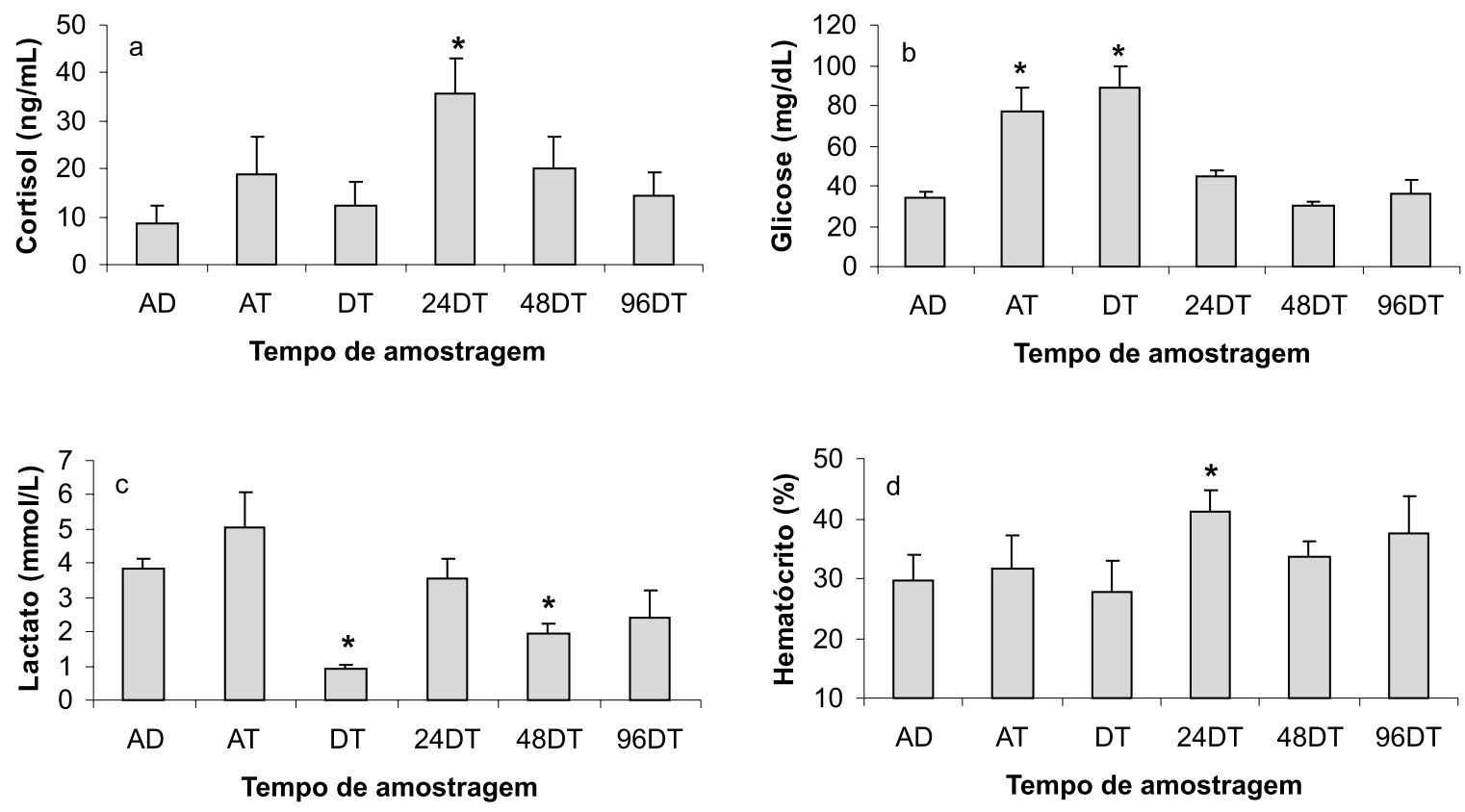

Figura 1- Cortisol (a), glicose (b), lactato (c) e hematócrito (d) de pirarucu (Arapaima gigas), antes e após o transporte em sacos plásticos por 3 horas. $\mathrm{AD}=$ antes do distúrbio; $\mathrm{AT}=$ antes do transporte; $\mathrm{DT}=$ depois do transporte; 24DT, 48DT e 96DT $=24$, 48 e 96 horas depois do transporte. * indica diferença significativa $(P<0,05)$ do controle $(A D)$ pelo teste Dunnett.

resposta. O aumento do hematócrito, como observado, indica uma hemoconcentração ocasionada pelo aumento da demanda de oxigênio, e tem a finalidade de auxíliar o peixe a retornar a situação de estresse (Morales et al., 2005). Para o tambaqui, não foram observadas alteraçōes no hematócrito, durante e após o transporte (Gomes et al., 2003a).

\section{EXPERIMENTO $\|$ - ADENSAMENTO}

$\mathrm{O}$ adensamento é, provavelmente, uma das etapas mais agressivas do manejo em uma piscicultura e se caracteriza pelo caráter agudo, severo e de curta duração. Sendo um procedimento que ocorre em determinadas situaçōes do cultivo intensivo como inspeção de rotina, biometria, seleção e transporte, nas quais uma série de procedimentos estressores é imposta aos animais (Urbinati $\&$ Carneiro, 2004). Neste experimento, o pequeno volume de água durante o adensamento obrigava os peixes a ficarem em contato permanente com o fundo da caixa, tendo consequentemente a natação comprometida. Neste período era possível observar o estresse abrasivo ocasionado pelos suscesivos encontros entre peixes e o intenso exercício físico ocasionado pela tentativa de natação. A abrasão mecânica e o exercício intenso entre peixes são tidas como importantes precursores de resposta de estresse (Ross \& Ross, 1999; Urbinati \& Carneiro, 2004).

Não houve registro de mortalidade durante o período do adensamento e até 48 horas após a realização do ensaio. Houve diferença significativa nas concentrações de cortisol plasmático, entre os tempos de amostragem DA e 24DA, quando comparados ao controle (AA). O valor médio do cortisol antes do adensamento foi de 23,8 $\pm 7,7 \mathrm{ng} / \mathrm{mL}$, e logo após o adensamento (DA), de $90,4 \pm 6,16 \mathrm{ng} / \mathrm{mL}$ (Figura 2a). As respostas de cortisol dos peixes submetidos ao adensamento foram mais agudas que as do transporte, pois ocorreu uma elevação significativa das concentrações logo após o estressor. Estudo realizado por Barnett \& Pankhurst (1998), com linguado (Rhombosolea tapirina), mostra que a elevação na concentração de cortisol após o adensamento permaneceu por até dois dias. A concentração de cortisol plasmático em pirarucu submetido ao adensamento retornou para valores semelhantes ao inicial 6 horas após ser submetido ao estressor. A glicose sanguínea apresentou um aumento significativo no tempo DA em relação ao controle (AA), retornando em seguida para valores basais. $\mathrm{O}$ valor médio da glicose no tempo DA foi 99,5 $\pm 3,2 \mathrm{mg} / \mathrm{dL}$. (Figura $2 \mathrm{~b}$ ).

O lactato apresentou um aumento significativo depois do adensamento $(10,9 \pm 0,6 \mathrm{mmol} / \mathrm{L})$, quando comparado com o momento controle $(2,18 \pm 0,3 \mathrm{mmol} / \mathrm{L}$ ) (Figura 2c). Esta grande elevação do lactato logo após o adensamento é reflexo do exercício que o peixe foi exposto, e já foi observado para outras espécies, como truta arco-íris (Onchorhynchus mykiss) (Wells \& Pankhurst, 1999) e o híbrido de esturjāo (Scaphirhynchus albus x $S$. platorynchus) (Barton et al., 2000). Os valores de lactato retornaram para valores iniciais 6 horas após o adensamento. Resultados obtidos por Barnett \& Pankhurst (1998) com 

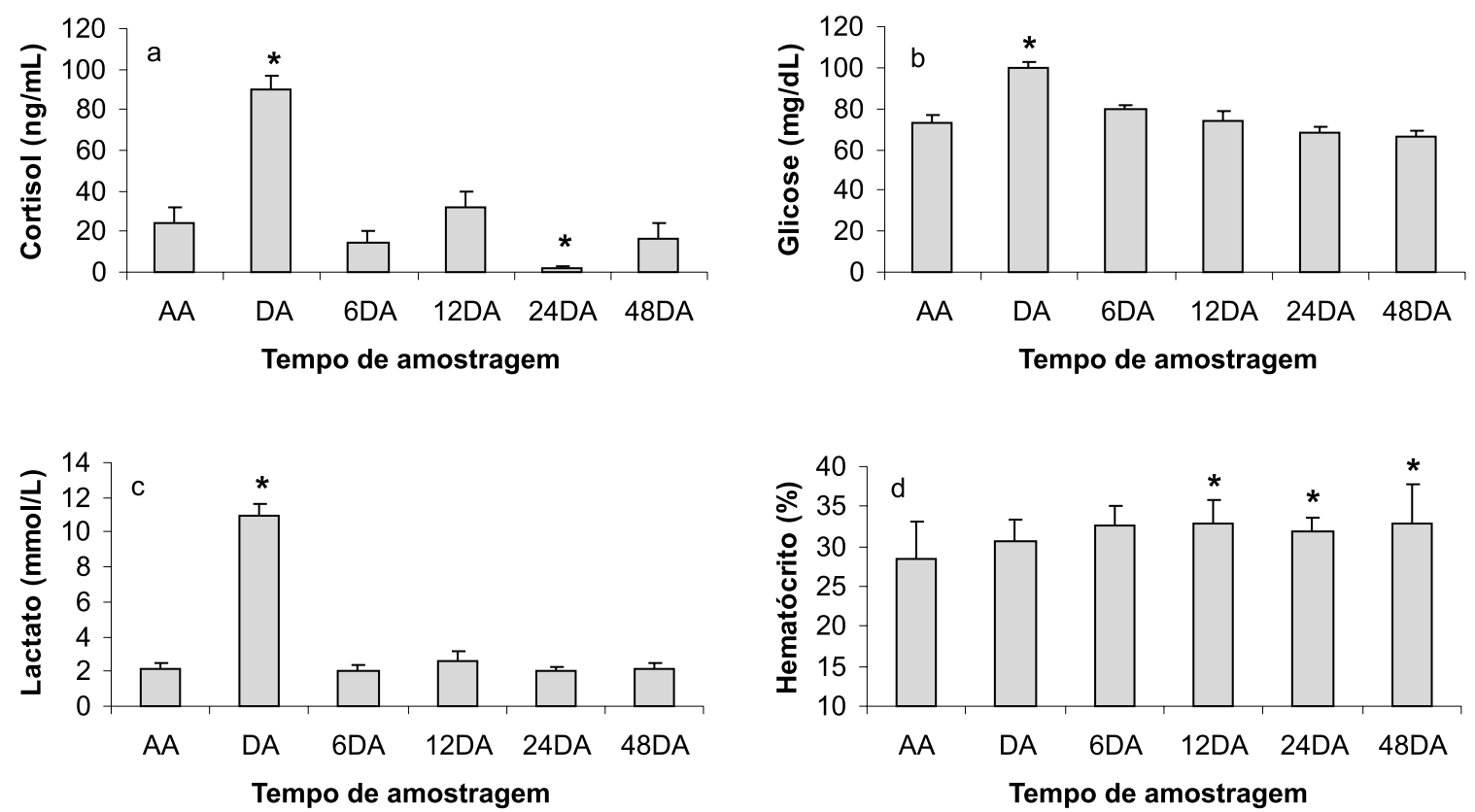

Figura 2 - Cortisol (a), glicose (b), lactato (c) e hematócrito (d) de pirarucu (Arapaima gigas), antes e após o adensamento por 30 minutos. $A A=$ antes do adensamento; $\mathrm{DA}=$ depois do adensamento; 6DA, 12DA, 24DA e 48DA =6, 12, 24 e 48 horas depois do adensamento. * indica diferença significativa $(\mathrm{P}<0,05)$ do controle $(\mathrm{AA})$, pelo teste de Dunnett.

linguado mostram um aumento nas concentrações de lactato após a exposição ao estressor, permanecendo por 48 horas. $\mathrm{O}$ hematócrito apresentou um aumento significativo nos tempos 12DA, 24DA e 48DA (Figura 2d). A principal explicação é o caráter mais agudo do estresse por adensamento, o que também foi refletido nos demais parâmetros analisados.

O cortisol, a glicose e o lactato apresentaram um padrão semelhante de resposta ao adensamento, havendo um aumento destes parâmetros no momento mais intenso de manejo (DA, logo após o adensamento). $\mathrm{O}$ rápido retorno à condição fisiológica inicial é favorável para a tomada de medidas preventivas, como uso de sal e outros profiláticos, que devem agir sobre o momento de maior intensidade de estresse.

\section{EXPERIMENTO III - EXPOSIÇÃO À AMÔNIA.}

Segundo Cavero et al. (2004), a principal fonte de compostos nitrogenados incorporados na água, na piscicultura intensiva, éa alimentação. No início da criação, quando as biomassas são menores, são observadas baixas concentraçôes de amônia, composto resultante do catabolismo das proteínas, que aumentam proporcionalmente à quantidade de alimento fornecido e ao aumento da biomassa (Hurvitz et al., 1997). No caso da criação de peixes carnívoros, como o pirarucu, esta situação pode ser agravada em virtude dos elevados níveis de proteínas usados nas rações. A exposição dos peixes a altas concentrações de amônia causa retardo no crescimento e torna os peixes mais susceptíveis às enfermidades como conseqüência do estresse (Moraes \& Martins, 2004).
Não houve mortalidade durante o período de exposição à amônia ou até 96 horas após a exposição. Os valores de cortisol plasmático não apresentaram diferença significativa nos diferentes tempos de amostragem quando comparado com o controle (Figura 3a). Person-le Ruyet et al. (2003), verificaram um rápido aumento no cortisol do turbot (Scophthalmus maximus) exposto a baixas concentraçóes de amônia $\left(0,17-0,34 \mathrm{mg} / \mathrm{L} \mathrm{NH}_{3}-\mathrm{N}\right)$, por outro lado, quando exposto a altas concentrações de amônia $\left(0,73 \mathrm{mg} / \mathrm{L} \mathrm{NH}_{3}-\mathrm{H}\right)$ o turbot apresenta uma lenta elevação do cortisol, atingindo o pico de resposta com 14 dias de exposição. Os resultados obtidos com pirarucu mostram um padrão de resposta diferente, uma vez, que não houve nenhuma resposta do cortisol nesta espécie, durante e após a exposição à amônia.

Houve diferença significativa nas concentrações de glicose sanguínea, no tempo de amostragem $96 \mathrm{DE}$, em relação ao controle. Os valores mais altos de glicose $(75,9 \pm 8,9 \mathrm{mg} / \mathrm{dL})$ foram obtidos logo após a exposição à amônia (DE) (Figura 3b). Após este tempo de amostragem a glicose diminuiu atingindo o valor mais baixo no tempo 96DE. Evans et al. (2006), observaram que a exposição da tilápia do Nilo (Oreochromis niloticus) a concentrações subletais de amônia, também ocasiona um aumento significativo nas concentraçôes de glicose, havendo, em seguida, uma diminuição gradual destes valores com o tempo de exposição.

O lactato apresentou diferença significativa nos tempos de amostragem 24DE, 48DE e 96DE quando comparados ao controle (Figura 3c). Houve uma diminuição do lactato ao longo 
das amostragens, sendo notória a diminuição do ritmo natação dos peixes, indicando uma resposta terciária de estresse, com a mudança do comportamento dos animais. Nenhuma alteração no hematócrito foi observada nos diferentes momentos de amostragem (Figura 3d).

O resultado obtido sugere que houve um esgotamento das reservas energéticas do peixe, pois mesmo em situação de estresse o pirarucu não foi capaz de aumentar a glicose sanguínea a fim de fornecer energia ao organismo para enfrentar a situação desfavorável.

\section{CONCLUSÕES}

- As respostas fisiológicas do pirarucu submetido aos três estressores são diferentes em sua intensidade e duração;

- O transporte e o adensamento induzem a maioria das respostas de estresse no pirarucu, sendo a magnitude de estresse do adensamento maior que a do transporte;

- O pirarucu quando exposto a altas concentraçóes de amônia apresenta uma grande latência nas respostas fisiológicas de estresse e tem pouca habilidade em mobilizar glicose, como energia, a fim de enfrentar a situação desfavorável.

- As alteraçôes metabólicas do pirarucu frente a um estressor, de forma geral, ocorrem no momento mais intenso de manejo, o que facilita minimizá-las com a adoção de boas práticas de manejo durante a criação da espécie.
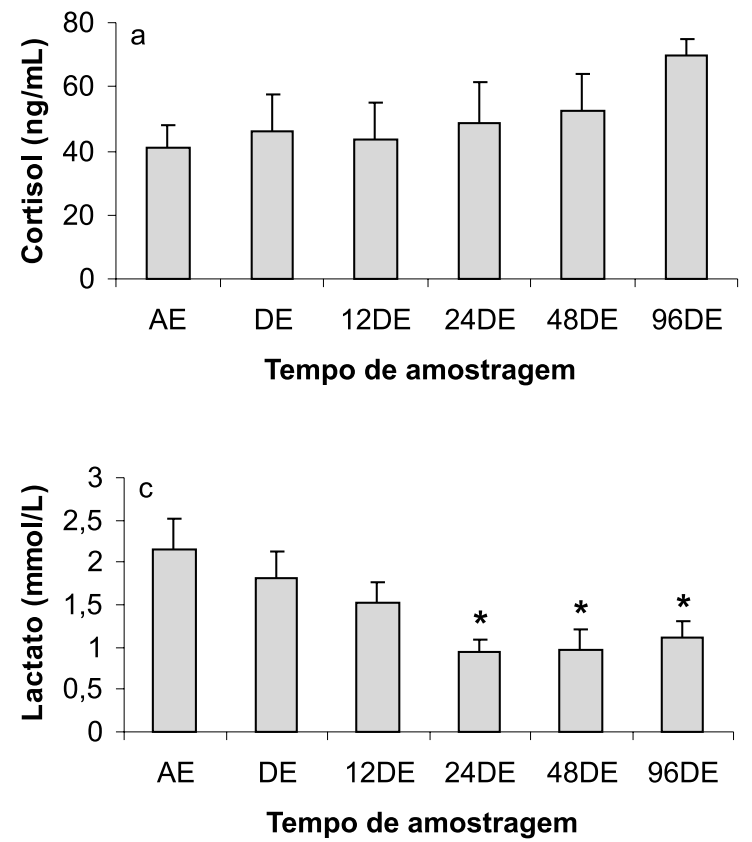

\section{AGRADECIMENTOS}

Aos estudantes e técnicos do laboratório de piscicultura da Embrapa Amazônia Ocidental pela ajuda nas diversas etapas deste trabalho. Ao senhor José Pereira de Souza pela alimentação dos peixes. Trabalho financiado pela Embrapa MP2 e pelo CNPq processos \# 475093/2003-8 e \# 506943/2004-6. LCG é bolsista de produtividade em pesquisa do CNPq.

\section{LITERATURA CITADA}

Barnett, C.W.; Pankhurst, N.W. 1998. The effects of common laboratory and husbandry practices on the stress response of greenback flounder Rhombosolea tapirina (Günther, 1862). Aquaculture. 162:313-329.

Barton, B.A. 1997. Stress in finfish: past, present and future a historical perspective. In: Iwama, G.K., Pickering, A.D., Sumpter, J.P., Schreck, C.B. (Eds.). Fish stress and health in aquaculture. Society for Experimental Biology Seminar Series 62. Cambridge University Press, New York, NY. p.1-33,

Barton, B.A. 2000. Salmonid fishes differ in their cortisol and glucose responses to handling and transport stress. North American Journal of. Aquaculture. 62:12-18.

Barton, B.A.; Bolling, H.; Hauskins, B.; Jansen, C.R. 2000. Juvenile pallid (Scaphirhynchus albuns) and hybrid pallid $\mathrm{x}$ shovelnose ( $S$. albuns $\mathrm{x} S$. platorynchus) sturgeons exhibit low physiological responses to acute handling and severe confinement. Comparative Biochemistry Physiology. 126A:125-134.
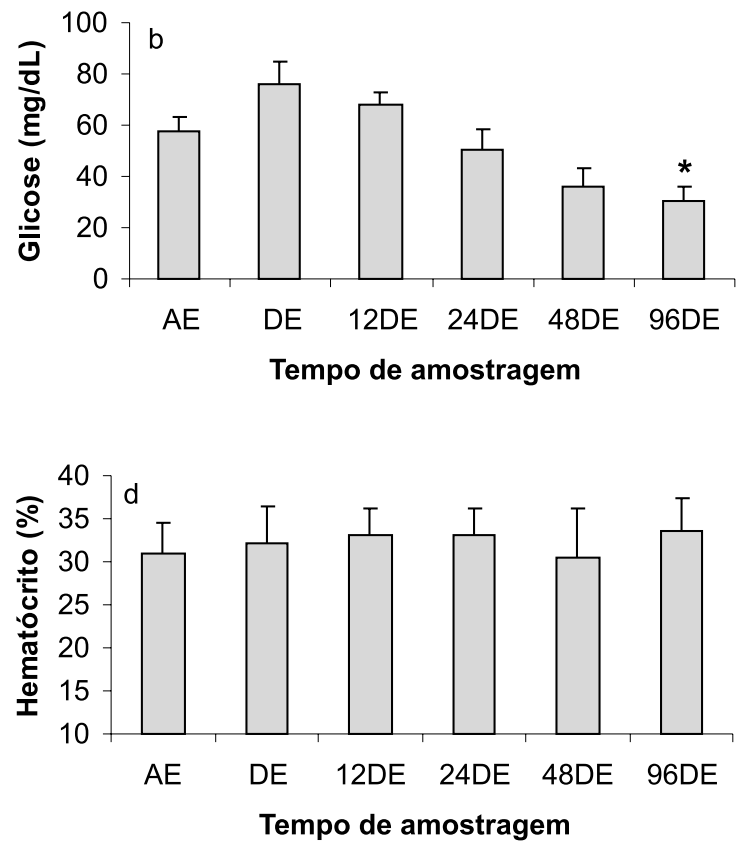

Figura 3 - Cortisol (a), glicose (b), lactato (c) e hematócrito (d) de pirarucu (Arapaima gigas), antes e após a exposição a 20 mg/L de amônia por 24 horas. $\mathrm{AE}=$ antes da exposição; $\mathrm{DE}=$ depois da exposição; 12DE, 24DE, 48DE e 96DE = 24, 48 e 96 horas depois da exposição. * indica diferença significativa $(\mathrm{P}<0,05)$ do controle $(\mathrm{AE})$, pelo teste de Dunnett. 
Barton, B.A.; Morgan, J.D.; Vijayan, M.M. 2002. Physiological and condition-related indicators of environmental stress in fish. In: Adams (ed.). Biological indicator of aquatic ecosystem stress, Bestherda, Maryland, American Fisheries Society, p.289-320.

Brinn, R.P. 2003. Cortisol exógeno em matrinxã (Brycon cephalus, Characidae, Günther, 1869): variáveis fisiológicas na maturação final e crescimento inicial da prole. Tese de Doutorado, UNESP. Jaboticabal, São Paulo 43p.

Carneiro, P.C.F.; Urbinati, E.C. 2001. Salt as a stress response mitigator of matrinxã, Brycon cephalus (Günther), during transport. Aquaculture Research, 32:298-307.

Cavero, B.A.S.; Pereira-Filho, M.; Bordinhon, A.M.; Fonseca, F.A.L.; Ituassú, D.R.; Roubach, R; Ono, E.A. 2004. Tolerância de juvenis de pirarucu ao aumento da concentração de amônia em ambiente confinado. Pesquisa Agropecuária Brasileira, 39:513516.

Cavero, B.A.S.; Pereira-Filho, M.; Roubach, R.; Ituassú, D.R.; Gandra, A.L. 2003. Efeito da densidade de estocagem na homogeneidade do crescimento de juvenis de pirarucu em ambiente confinado. Pesquisa Agropecuária Brasileira, 38:103107.

Evans, J.J.; Pasnik, D.J.; Brill, G.C.; Klesius, P.H. 2006. Un-ionized ammonia exposure in Nile Tilapia: toxicity, stress response, and susceptibility to Streptococcus agalactiae. North American Journal of Aquaculture, 68(1):23-33.

Ferraris Jr., C.J. 2003. Arapaimidae. In: Reis, R.E.; Kullander, S.O.; Ferraris, Jr, C.J. (Eds.). Check list of the freshwater fishes of South and Central America. Edipucrs, Porto Alegre, p. 31.

Gomes, L.C. 2002. Transporte de juvenis de tambaqui Colossoma macropomum (CUVIER 1818) (Teleostei, Characidade). Tese de Doutorado, Instituto Nacional de Pesquisas da Amazônia/ Fundação Universidade do Amazonas. Manaus, 101p.

Gomes, L.C.; Araujo-Lima, C.A.R.M.; Roubach, R., ChippariGomes, A.R.; Lopes, N.P. 2003a. Effect of fish density during transportation on stress and mortality of juvenile tambaqui Colossoma macropomum. Journal of the World Aquaculture Society, 34:76-84

Gomes, L. C.; Roubach, R.; Cavero, B. A. S.; Pereira-Filho, M; Urbinati, E.C. 2003b Transport of pirarucu Arapaima gigas juveniles in plastic Bag. Acta Amazonica, 33:631-636.

Hurvitz, A.; Bercovier, H.; Rijn, J.V. 1997. Effect of ammonia on the survival and the immune response of rainbow trout (Oncorhynchus mykiss, Walbaum) vaccinated against Streptococcus iniae. Fish \& Shellfish Immunology, 7:45-53.

Imbiriba, E.P. 2001. Potencial da criação de pirarucu, Arapaima gigas, em cativeiro. Acta Amazonica. 31:299-316.

Iversen, M.; Finstad, B.; Nilssen, K.J. 1998. Recovery from loading and transport stress in Atlantic salmon (Salmo salar L.) smolts. Aquaculture. 168:387-394.

Kriger-Azolini, M.H.; Carosfeld, J.; Delattre, E.; Ceccarelli, P.S. 1989. Determinação dos indicadores endócrinos e metabólicos no estresse no manejo em pacu juvenil, Piaractus mesopotamicus
Homlberg, Boletim Técnico do CEPTA, 2:35-42.

Moraes, F.R.; Martins, M.L. 2004. Condiçôes predisponentes e principais enfermidades de teleósteos em piscicultura intensiva. In: Cyrino, J.E.P.; Urbinati, E.C.; Fracalossi, D.M.; Castagnolli, N. (Eds.). Tópicos especiais em piscicultura de água doce tropical intensiva. Sociedade Brasileira de Aqüicultura e biologia Aquática. Editora Tecart, São Paulo, p. 343-386.

Mommsen, T.P.; Vijayan, M.M.; Moon, T.W. 1999. Cortisol in teleosts: dynamics, mechanisms of action, and metabolic regulation. Reviews in Fish Biology and Fisheries, 9:211-268.

Morales, A.E.; Cardenete, G.; Abellán, E.; García-Rejón, L., 2005. Stress-related physiological responses to handling in common dentex (Dentex dentex Linnaeus, 1758). Aquaculture Research, 36:33-40.

Morgan, J.D.; Iwama, G.K. 1997. Measurements of stressed states in the field. In: Iwama, G.K., Pickering, A.D., Sumpter, J.P., Schreck, C.B. (Eds.). Fish stress and health in aquaculture. Society for Experimental Biology Seminar Series 62. Cambridge University Press, New York, NY. p. 247-270.

Pereira-Filho M.; Cavero, B.A.S.; Roubach, R.; Ituassú, D.R.; Gandra; A.L.; Crescêncio, R. 2003. Cultivo do Pirarucu (Arapaima gigas) em viveiro escavado. Acta Amazonica, 33:715718.

Person-le Ruyet, J.; Lamers A.; le Roux A.; Sévère, A.; Boeuf G.; Mayer-Gostan N. 2003. Long-term ammonia exposure of turbot: effects on plasma parameters. Journal of Fish Biology, 62:879-894.

Ross, L.G.; Ross, B. 1999. Anaesthethic and sedative techniques for aquatic animals. Blackwell Science, Oxford. 159p.

Salvo-Souza, R. H.; Val, A. L. 1990. O gigante das águas doces. Ciência Hoje, 11:9-12.

Urbinati, E.C.; Abreu, J.S.; Carmargo, A.C.S.; Landines, M.A. 2004. Loading and transport stress in juveniles matrinxã (Brycon cephalus) at various densities. Aquaculture, 229:389-400.

Urbinati, E.C.; Carneiro, P.C.F. 2004. Práticas de manejo e estresse dos peixe em piscicultura. In: Cyrino, J.E.P.; Urbinati, E.C.; Fracalossi, D.M.; Castagnolli, N. (Eds.). Tópicos especiais em piscicultura de água doce tropical intensiva. Sociedade Brasileira de Aqüicultura e biologia Aquática. Editora Tecart, São Paulo, p. 171-193.

Wells, R.M.G.; Pankhurst, N.W. 1999. Evaluation of simple instruments for the measurement of blood glucose and lactate, and plasma protein a stress indicator in fish. Journal of the World Aquaculture Society. 30:276-284.

Wedemeyer, G.A. 1996. Physiology of fish in intensive culture systems. Chapman and Hall, New York. 232 p.

Recebido em 09/03/2005

Aceito em 22/08/2006 
